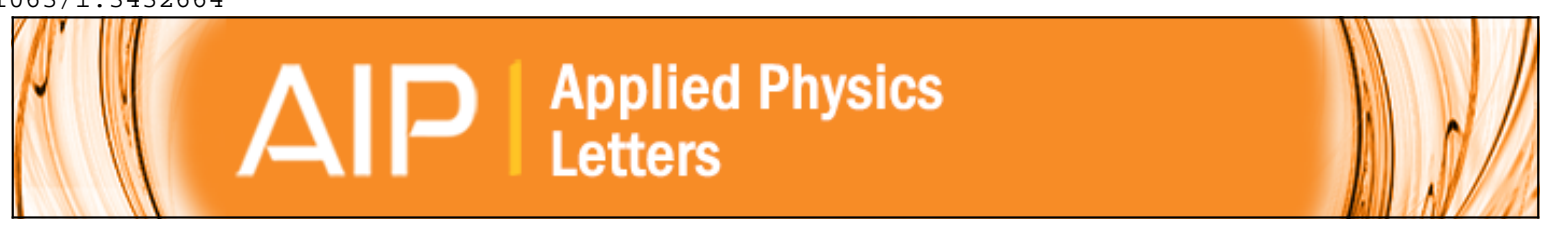

\title{
Ion transport and storage of ionic liquids in ionic polymer conductor network
}

\section{composites}

Yang Liu, Sheng Liu, Junhong Lin, Dong Wang, Vaibhav Jain, Reza Montazami, James R. Heflin, Jing Li, Louis Madsen, and Q. M. Zhang

Citation: Applied Physics Letters 96, 223503 (2010); doi: 10.1063/1.3432664

View online: http://dx.doi.org/10.1063/1.3432664

View Table of Contents: http://scitation.aip.org/content/aip/journal/apl/96/22?ver=pdfcov

Published by the AIP Publishing

\section{Over 700 papers \& presentations on} multiphysics simulation 


\title{
Ion transport and storage of ionic liquids in ionic polymer conductor network composites
}

\author{
Yang Liu, ${ }^{1}$ Sheng Liu, ${ }^{1}$ Junhong Lin, ${ }^{2}$ Dong Wang, ${ }^{3}$ Vaibhav Jain, ${ }^{4}$ Reza Montazami, ${ }^{5}$ \\ James R. Heflin, ${ }^{3}$ Jing Li, ${ }^{6}$ Louis Madsen, ${ }^{6}$ and Q. M. Zhang ${ }^{1,2, a)}$ \\ ${ }_{1}^{1}$ Department of Electrical Engineering, Pennsylvania State University, University Park, \\ Pennsylvania 16802, USA \\ ${ }^{2}$ Department of Materials Science and Engineering, Pennsylvania State University, University Park, \\ Pennsylvania 16802, USA \\ ${ }^{3}$ Department of Physics, Virginia Tech, Blacksburg, Virginia 24061, USA \\ ${ }_{5}^{4}$ Macromolecular Science and Engineering, Virginia Tech, Blacksburg, Virginia 24061, USA \\ ${ }^{5}$ Department of Materials Science and Engineering, Virginia Tech, Blacksburg, Virginia 2406, USA \\ ${ }^{6}$ Department of Chemistry, Virginia Tech, Blacksburg, Virginia 24061, USA
}

(Received 24 February 2010; accepted 16 April 2010; published online 2 June 2010)

\begin{abstract}
We investigate ion transport and storage of ionic liquids in ionic polymer conductor network composite electroactive devices. Specifically, we show that by combining the time domain electric and electromechanical responses, one can gain quantitative information on transport behavior of the two mobile ions in ionic liquids (i.e., cation and anion) in these electroactive devices. By employing a two carrier model, the total excess ions stored and strains generated by the cations and anions, and their transport times in the nanocomposites can be determined, which all depend critically on the morphologies of the conductor network nanocomposites. (C) 2010 American Institute of Physics. [doi:10.1063/1.3432664]
\end{abstract}

Ionic liquids (ILs), due to many unique and attractive features as electrolytes, have shown a great promise for applications in energy storage, conversion devices, and other electroactive devices. ${ }^{1-11}$ For example, the negligible vapor pressure of ILs enables these electroactive devices to operate at ambient atmosphere with long life cycles; the wide electrochemical window allows these devices to work at higher voltage; and the high ion mobility can lead to fast device response time. All of these properties are highly desirable for electroactive devices such as ionic polymer actuators, $1,5-8$ supercapacitors, ${ }^{2}$ batteries, ${ }^{10}$ fuel cells, ${ }^{11}$ and dye-sensitized solar cells. ${ }^{9}$ Figure 1 presents schematically a typical device configuration for such an electroactive device, which basically has a three-layer structure. The two porous composite electrodes separated by an ion conducting membrane provide high specific electrode area for ion storage under an applied voltage while allowing easy ion transport. One critical issue for ILs in these electroactive devices is the transport process, where fast transport speed is highly desirable. Moreover, the strain generated by the mobile ions in these devices is also of great concern.

This letter investigates the transport and storage behaviors of ILs in electroactive devices, referred to as the ionic polymer conductor network composites (IPCNCs), as illustrated in Fig. 1. Distinct from traditional electrolytes, ILs are salts in the liquid state with low bonding energy between the cation and anion, which leads to high ion concentration and ionic conductivity. A challenge in the device characterization is how to distinguish transport and other electroactive properties of the two different ions in a composite device configuration. Here we show that by combining the time domain electrical impedance and electromechanical response, one can gain quantitative information of the ion transport and storage in these devices.

A broad range of IPCNCs were selected for the study, which include IPCNCs fabricated with Nafion ${ }^{\mathrm{TM}}$ and Aquivion $^{\mathrm{TM}}$ ionomers via the direct assembly method ${ }^{6,7,12}$ and IPCNCs by the layer-by-layer (LbL) self-assembly method. ${ }^{8,13}$ Two ILs were examined as electrolytes, where 1-ethyl-3-methylimidazolium $\left(\left[\mathrm{EMI}^{+}\right]\right)$served as the cation and tetrafluoroborate $\left(\left[\mathrm{BF}_{4}^{-}\right]\right)$and trifluoromethanesulfonate $\left(\left[\mathrm{Tf}^{-}\right]\right)$served as the anions. ${ }^{14}$

For the IPCNCs fabricated with the directly assembly method, either the commercial Nafion film NR-211 of thickness $25 \mu \mathrm{m}$ or Aquivion from Solvay was chosen as the spacer layer in Fig. 1. $\mathrm{RuO}_{2} /$ Nafion (or $\mathrm{RuO}_{2} /$ Aquivion) composite was fabricated by mixing $\mathrm{RuO}_{2}$ nanoparticles (from Alfa Aesar with 13-19 nm diameter) with 20\% Nafion dispersion from Aldrich (or 20\% Aquivion dispersion from Solvay). In this study, a CNC layer with 40 vol \% of $\mathrm{RuO}_{2}$ nanoparticles was used. IPCNCs fabricated by the LbL method were made by alternately immersing the Nafion film into two aqueous solutions containing the polycation poly(allylamine hydrochloride) (PAH) as the polyanion and polycation and anionic gold nanoparticles $(2 \mathrm{~nm}$, from Purest Colloids) or CNT (from Carbon Solutions Inc.). ${ }^{8,14}$ The LbL CNCs comprise 100 bilayers of PAH/Au and 30 bilayers of $\mathrm{PAH} / \mathrm{CNT}$, respectively. The lateral dimensions of the IPC-

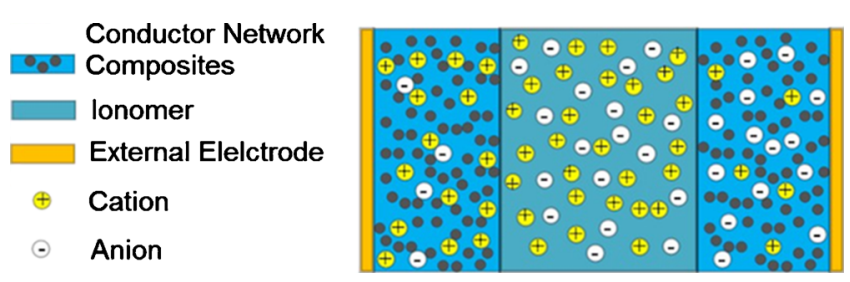




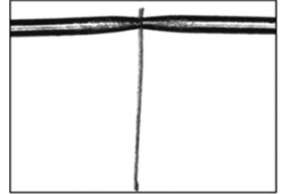

(a) $t=0$

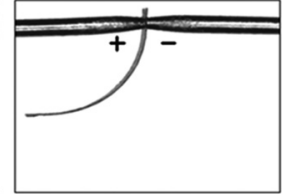

(b) $\mathrm{t}=\mathrm{t}_{1}$

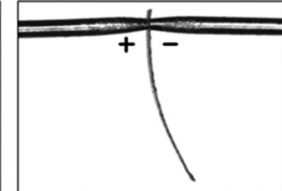

(c) $t=t_{2}$
FIG. 2. The bending actuation recorded at different times $\left(t_{2}>t_{1}\right)$ for the $\mathrm{PAH} / \mathrm{Au}$ IPCNCs under $4 \mathrm{~V}$ step voltage.

NCs studied here are $1 \times 10 \mathrm{~mm}^{2}$. The thickness ranges approximately from $26 \mu \mathrm{m}$ for LbL IPCNCs to $31 \mu \mathrm{m}$ for the IPCNCs made from the directly assembly method.

A potentiostat (Princeton Applied Research 2273) was utilized for electrical measurement. In this study, a dc step voltage is applied on the IPCNC samples and the charging current I versus time was recorded and integrated to obtain the stored charge versus time at the CNC electrodes. The time resolved electromechanical response of the actuators was recorded by a probe station (Cascade Microtech M150) equipped with a Leica microscope and a charge-coupled device camera (Pulnix 6740CL). Nuclear magnetic resonance (NMR) provided quantitative measures of self-diffusion coefficients for IL anions ( ${ }^{19} \mathrm{~F}$ NMR) and cations $\left({ }^{1} \mathrm{H}\right.$ NMR) both in pure ILs and in ILs absorbed into ionomers. ${ }^{15}$ Ion mobility was calculated from diffusion coefficients using the Einstein relation $\mu=D e / k_{B} T$.

Presented in Fig. 2 is the evolution of the bending actuation of the PAH/Au IPCNCs under $4 \mathrm{~V}$ step voltage. The actuator initially bends toward the anode and as the time progresses; it reverses the actuation direction and bends toward the cathode. As will be discussed later, this is caused by the two mobile ions in the actuation process. The intrinsic strain $\mathrm{S}_{0}$ generated in the composite electrode $(\mathrm{CNC})$ layer is proportional to the bending curvature $\kappa(\kappa=1 / \mathrm{R}, \mathrm{R}$ is the radius of curvature), as shown in Fig. $3{ }^{8,14}$ The data shows that for all the IPCNCs studied, the cathode expands at first and then the strain decreases ( $\kappa$ becomes small). For the data in Figs. 3(b)-3(d), the strain at cathode becomes negative ( $\kappa$ changes sign). Figure 3 also presents the charge density $Q$ versus time for these IPCNCs.

The results in Figs. 2 and 3 can be understood based on a two-carrier model to account for the presence of the mobile cations and anions in the IPCNCs which have different mobility. Using a simplified RC equivalent circuit model for each type of ion (cations and anions) in the IPCNCs lead to Fig. 4(a) where the two branches represent the ion transport of two types of ions. In the figure, the RC transmission line equivalent circuits used in the literature for the porous electrode in each branch are simplified into $\mathrm{R}_{\text {cat }}$ and $\mathrm{C}_{\text {cat }}$ or $\mathrm{R}_{\mathrm{an}}$ and $\mathrm{C}_{\mathrm{an}}$, respectively, where the subscripts "cat" and "an" indicate cations and anions. ${ }^{16}$ Hence, the charge density $Q$ versus time under a step voltage becomes

$$
Q(t)=Q_{0}^{\mathrm{cat}}\left(1-e^{-t / \tau_{\mathrm{cat}}}\right)+Q_{0}^{\mathrm{an}}\left(1-e^{-t / \tau_{\mathrm{an}}}\right),
$$

where the time constant $\tau_{\text {cat }}$ and $\tau_{\text {an }}$ will depend on the porous electrode morphology as well as the ion mobility (=RC time constant).

The accumulation or depletion of excess ions in the composite electrodes will cause expansion and contraction in the porous electrode regions. As schematically illustrated in Fig. 4(b), in contrast to the conduction current in which the
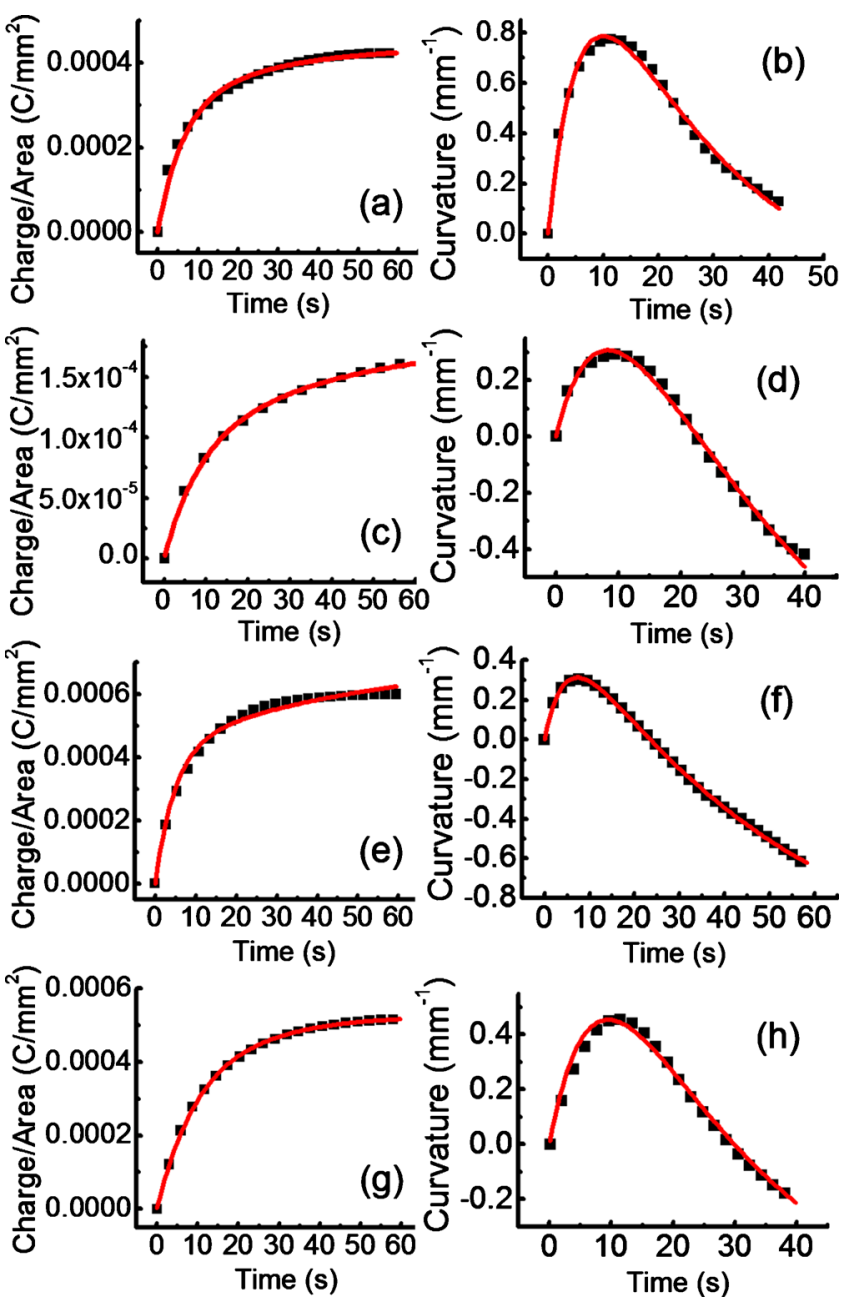

FIG. 3. (Color online) Data and fitting of charge and curvature vs time for the IPCNCs: (a) charge and (b) curvature for Nafion with $3 \mu \mathrm{m}$ thick Nafion $/ \mathrm{RuO}_{2}$ composite at each side and $\mathrm{EMIBF}_{4} \mathrm{IL}$ under $3 \mathrm{~V}$ step voltage; (c) charge and (d) curvature for Aquivion with $3 \mathrm{um}$ Aquivion/ $\mathrm{RuO}_{2}$ composite and EMITf IL under $3 \mathrm{~V}$ step voltage; (e) charge and (f) curvature for Nafion with $180 \mathrm{~nm}$ PAH/Au LbL composite and EMITf IL under $4 \mathrm{~V}$ step voltage; and (g) charge and (h) curvature for Nafion with $400 \mathrm{~nm}$ PAH/CNT LbL composite and EMITf IL under $4 \mathrm{~V}$ step voltage.

oppositely charged mobile ions add together [Eq. (1)], the strains they generate will have opposite sign and hence will cancel each other. Therefore, the curvature, which is proportional to the net strain in the IPCNCs, follows as:

$$
\kappa(t)=\kappa_{0}^{\mathrm{cat}}\left(1-e^{-t / \tau_{\mathrm{cat}}}\right)-\kappa_{0}^{\mathrm{an}}\left(1-e^{-t / \tau_{\mathrm{an}}}\right) .
$$

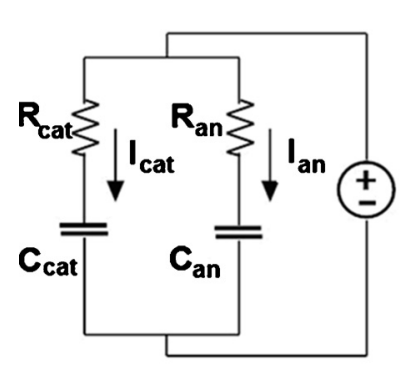

(a)

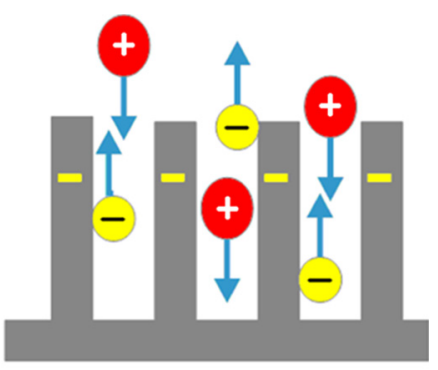

(b)
FIG. 4. (Color online) (a) Equivalent circuit with two carrier currents. (b) Schematic ilustration of strain cancellation effect in electrodes 
TABLE I. Summary of fitting parameters for the IPCNCs studied.

\begin{tabular}{lcccc}
\hline \hline & $\begin{array}{c}\mathrm{RuO}_{2} / \mathrm{Nafion} \\
\mathrm{EMIBF}_{4}\end{array}$ & $\begin{array}{c}\mathrm{RuO}_{2} / \text { Aquivion } \\
\text { EMITf }\end{array}$ & $\begin{array}{c}\text { PAH/Au LbL } \\
\text { EMITf }\end{array}$ & $\begin{array}{c}\text { PAH/CNT LbL } \\
\text { EMITf }\end{array}$ \\
\hline$\tau^{\text {cat }}(\mathrm{s})$ & 6 & 7.9 & 4.83 & 9 \\
$\tau^{\text {an }}(\mathrm{s})$ & 25 & 36.3 & 53 & 20 \\
$Q^{\text {cat }}\left(\mathrm{C} / \mathrm{mm}^{2}\right)$ & $2.82 \times 10^{-4}$ & $8.46 \times 10^{-5}$ & $4.28 \times 10^{-4}$ & $3.17 \times 10^{-4}$ \\
$Q^{\text {an }}\left(\mathrm{C} / \mathrm{mm}^{2}\right)$ & $1.57 \times 10^{-4}$ & $9.48 \times 10^{-5}$ & $2.91 \times 10^{-4}$ & $2.10 \times 10^{-4}$ \\
$\kappa^{\text {cat }}\left(\mathrm{mm}^{-1}\right)$ & 1.83 & 1.29 & 0.74 & 2.5 \\
$\kappa^{\text {an }}\left(\mathrm{mm}^{-1}\right)$ & 2.13 & 2.62 & 2.04 & 3.1 \\
\hline \hline
\end{tabular}

Equations (1) and (2) are used to fit the experimental data (solid curves in Fig. 3) and the fitting parameters are summarized in Table I. The fitting results indicate that $\tau^{\text {cat }}$ is smaller than $\tau^{\text {an }}$. That is, the cations have fast transport speed compared with the anions, which causes the initial bending actuation from the expansion of cathode and contraction of the anode. As time progresses, the anions will generate strain in the opposite direction and eventually cause the IPCNCs to bend in the opposite direction. $\tau_{\text {cat }}$ and $\tau_{\text {an }}$, as well as the ratio of $\tau_{\text {an }} / \tau_{\text {cat }}$ depend highly on the electrode morphology and the coupling between the ions and the composite electrodes. $\tau^{\text {an }} / \tau^{\text {cat }}$ for the IPCNCs with $\mathrm{RuO}_{2} /$ Nafion $(=4.16)$ and $\mathrm{RuO}_{2}$ / Aquivion $(=4.6)$ are similar. For the IPCNCs with $\mathrm{LbL}$ electrode layers, $\tau^{\mathrm{an}} / \tau^{\mathrm{cat}}$ is 10.97 for $\mathrm{PAH} / \mathrm{Au}$ and 2.22 for PAH/CNT, respectively.

For comparison, the diffusion coefficient from the NMR data and the mobility deduced from the Einstein relation for neat ILs and the EMI-Tf IL in Nafion (NR-212) are listed in Table II. Notably, the cation $\left(\mathrm{EMI}^{+}\right)$has higher mobility than that of anions $\left(\mathrm{BF}_{4}^{-}\right.$and $\left.\mathrm{Tf}^{-}\right)$, which is consistent with the experimental results presented s. The ratio of $\mu_{\text {cat }} / \mu_{\text {an }}$ is 1.27 in neat $\mathrm{EMI}^{+} \mathrm{BF}_{4}^{-}$and 1.5 in neat $\mathrm{EMI}^{+} \mathrm{Tf}^{-}$, which are much smaller than the ratio of $\tau_{\text {an }} / \tau_{\text {cat }}$ from the ion transport in the IPCNCs. These results indicate the crucial role played by the CNC morphology in determining the ion transport in the porous electrodes. Moreover, the large increase in $\tau_{\text {an }}$ compared with $\tau_{\text {cat }}$ suggests the possible large size (ions plus the solvent or aggregation shells) of the anions in the transport process in these composites electrodes.

A comparison of the ratio of $\kappa^{\text {an }} / Q^{\text {an }}$ with $\kappa^{\text {cat }} / Q^{\text {cat }}$ reveals that the anions are more effective in generating strain

TABLE II. Diffusion coefficients (measured) and electrophoretic mobilities (calculated) from pulsed-gradient NMR for the ILs studied.

\begin{tabular}{|c|c|c|c|c|c|c|}
\hline & \multicolumn{2}{|c|}{$\mathrm{EMIBF}_{4}$} & \multicolumn{2}{|c|}{ EMITf } & \multicolumn{2}{|c|}{ EMITf in Nafion ${ }^{a}$} \\
\hline & $\mathrm{EMI}^{+}$ & $\mathrm{BF}_{4}^{-}$ & $\mathrm{EMI}^{+}$ & $\mathrm{Tf}^{-}$ & $\mathrm{EMI}^{+}$ & $\mathrm{Tf}^{-}$ \\
\hline$D\left(10^{-12} \mathrm{~m}^{2} \mathrm{~s}^{-1}\right)$ & 43.0 & 33.7 & 44.0 & 29.2 & 3.86 & 2.73 \\
\hline$\mu\left(10^{-9} \mathrm{~m}^{2} \mathrm{~V}^{-1} \mathrm{~s}^{-1}\right)$ & 1.67 & 1.31 & 1.71 & 1.14 & 0.150 & 0.106 \\
\hline
\end{tabular}

32 wt \% EMITf in Nafion with 2.1 wt \% coexisting water. All data are taken at $25{ }^{\circ} \mathrm{C}$. in these porous electrodes. This again suggests that the anions when they are stored in the porous electrodes have much larger solvent or aggregation shells, or that they associate more strongly with the ionic electrode matrix, resulting in a larger effective ion size and higher strain generated. The results here demonstrate a simple means to compare the effective ion size of the cation and anion of an IL in electroactive devices.

The authors thank Ralph Colby and Wenjuan Liu for discussions. This material is based upon work supported in part by the U.S. Army Research Office under Grant No. W911NF-07-1-0452, Ionic Liquids in Electro-Active Devices (ILEAD) MURI, and by NSF under the Grant No. CMMI 0709333.

${ }^{1}$ W. Lu, A. G. Fadeev, B. H. Qi, E. Smela, B. R. Mattes, J. Ding, G. M. Spinks, J. Mazurkiewicz, D. Z. Zhou, G. G. Wallace, D. R. MacFarlane, S. A. Forsyth, and M. Forsyth, Science 297, 983 (2002).

${ }^{2}$ M. Ue, M. Takeda, A. Toriumi, A. Kominato, R. Hagiwara, and Y. Ito, J. Electrochem. Soc. 150, A499 (2003).

${ }^{3}$ M. Galinski, A. Lewandowski, and I. Stepniak, Electrochim. Acta 51, 5567 (2006).

${ }^{4}$ S. Ono, S. Seki, R. Hirahara, Y. Tominan, and J. Takeya, Appl. Phys. Lett. 92, 103313 (2008).

${ }^{5}$ Y. Bar-Cohen and Q. M. Zhang, MRS Bull. 33, 173 (2008).

${ }^{6}$ D. Kim, K. J. Kim, Y. Tak, D. Pugal, and I.-S. Park, Appl. Phys. Lett. 90, 184104 (2007).

${ }^{7}$ M. D. Bennett and D. J. Leo, Sens. Actuators, A 115, 79 (2004).

${ }^{8}$ S. Liu, R. Montazami, Y. Liu, V. Jain, M. Lin, J. R. Heflin, and Q. M. Zhang, Appl. Phys. Lett. 95, 023505 (2009).

${ }^{9}$ P. Wang, B. Wenger, R. Humphry-Baker, J. E. Moser, J. Teuscher, W. Kantlehner, J. Mezger, E. V. Stoyanov, S. M. Zakeeruddin, and M. Gratzel, J. Am. Chem. Soc. 127, 6850 (2005).

${ }^{10}$ N. Byrne, P. C. Howlett, D. R. MacFarlane, and M. Forsyth, Adv. Mater (Weinheim, Ger.) 17, 2497 (2005).

${ }^{11}$ R. F. de Souza, J. C. Padilha, R. S. Goncalves, and J. Dupont, Electrochem. Commun. 5, 728 (2003).

${ }^{12}$ J. Akle, D. Leo, M. Hickner, and J. McGrath, J. Mater. Sci. 40, 3715 (2005)

${ }^{13}$ V. Jain, H. M. Yochum, R. Montazami, and J. R. Heflin, Appl. Phys. Lett. 92, 033304 (2008).

${ }^{14}$ S. Liu, R. Montazami, Y. Liu, V. Jain, M. Lin, X. Zhou, J. R. Heflin, and Q. M. Zhang, Sens. Actuators, A 157, 267 (2010).

${ }^{15}$ J. Li, K. G. Wilmsmeyer, J. B. Hou, and L. A. Madsen, Soft Matter 5, 2596 (2009)

${ }^{16}$ B. E. Conway and W. G. Pell, J. Solid State Electrochem. 7, 637 (2003). 